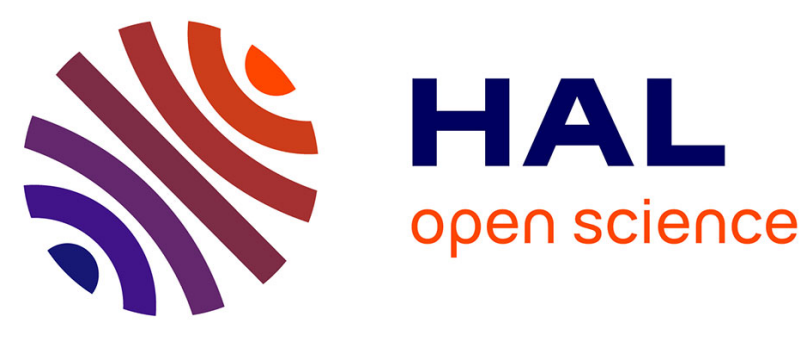

\title{
New concept for immediate breast reconstruction for invasive cancers: feasibility, oncological safety and esthetic outcome of post-neoadjuvant therapy immediate breast reconstruction versus delayed breast reconstruction: a prospective pilot study
}

Pierre Ludovic Giacalone, Gauthier Rathat, Jean Pierre Daures, Paul Benos, David Azria, Caroline Rouleau

\section{- To cite this version:}

Pierre Ludovic Giacalone, Gauthier Rathat, Jean Pierre Daures, Paul Benos, David Azria, et al.. New concept for immediate breast reconstruction for invasive cancers: feasibility, oncological safety and esthetic outcome of post-neoadjuvant therapy immediate breast reconstruction versus delayed breast reconstruction: a prospective pilot study. Breast Cancer Research and Treatment, 2010, 122 (2), pp.439-451. 10.1007/s10549-010-0951-7 . hal-00540028

\section{HAL Id: hal-00540028 \\ https://hal.science/hal-00540028}

Submitted on 26 Nov 2010

HAL is a multi-disciplinary open access archive for the deposit and dissemination of scientific research documents, whether they are published or not. The documents may come from teaching and research institutions in France or abroad, or from public or private research centers.
L'archive ouverte pluridisciplinaire HAL, est destinée au dépôt et à la diffusion de documents scientifiques de niveau recherche, publiés ou non, émanant des établissements d'enseignement et de recherche français ou étrangers, des laboratoires publics ou privés. 


\title{
New concept for immediate breast reconstruction for invasive cancers: feasibility, oncological safety and esthetic outcome of post-neoadjuvant therapy immediate breast reconstruction versus delayed breast reconstruction: a prospective pilot study
}

\author{
Pierre Ludovic Giacalone - Gauthier Rathat · Jean Pierre Daures • \\ Paul Benos · David Azria · Caroline Rouleau
}

Received: 9 February 2010/ Accepted: 12 May 2010/Published online: 26 May 2010

(C) Springer Science+Business Media, LLC. 2010

\begin{abstract}
Feasibility and oncological safety of postadjuvant skin-sparing mastectomy (SSM) plus immediate breast reconstruction (IBR) cannot be evaluated by randomized trials. However, comparative study could modify guidelines for the oncosurgical treatment of invasive breast cancer. Our study compared the feasibility, oncological safety and esthetic outcome of SSM plus latissimus dorsi (LD) flap IBR after chemotherapy (CT) and radiotherapy (RT) with the standard management for invasive breast cancer: mastectomy as primary treatment, adjuvant $\mathrm{CT}$ and $\mathrm{RT}$, and LD flap delayed breast reconstruction (DBR). Twenty-six selected patients with stages IIA-IIIA breast cancer were offered post-neoadjuvant SSM plus IBR with LD flap plus implant (IBR group). Seventy-eight other patients had primary mastectomy, adjuvant $\mathrm{CT}$ and RT, and LD-assisted DBR (DBR group). After 4.1 years (range 1-8) of follow-up, feasibility, oncological safety, and esthetic outcome were compared. Sixteen (61\%) early complications were reported for the IBR group versus 44 (56\%) for the DBR group $(P=0.645)$. Early implant loss
\end{abstract}

P. L. Giacalone $(\bowtie) \cdot$ G. Rathat · P. Benos

Oncology Unit, Department of Obstetrics and Gynecology,

Hôpital Arnaud de Villeneuve, 371 rue du Doyen Gaston Giraud,

34295 Montpellier Cedex 5, France

e-mail: pl.giacalone@cegetel.net

\section{J. P. Daures}

Biostatistics Unit, Clinical Research Center, 75 rue de la Cardonille, 34295 Montpellier Cedex 5, France

D. Azria

Centre Val d'Aurelle, 34000 Montpellier, France

C. Rouleau

Department of Pathology, Hôpital Lapeyronie, 371 rue du Doyen

Gaston Giraud, 34295 Montpellier Cedex 5, France was $0 \%$ in IBR versus $12 \%$ in DBR. IBR had $8(30 \%)$ late complications versus $17(21 \%)$ for DBR $(P=0.362)$. Capsular contracture and reconstruction failure rates were similar. Local recurrence was $7.7 \%$ (2/26) in IBR and $6.4 \%$ $(5 / 78)$ in DBR $(P=0.823)$. Cosmetic evaluation by independent physicians and by the patients themselves was identical in the two groups. Our concept provides a basis for offering more women the opportunity to elect for immediate reconstruction, even in the setting of radiation therapy.

Keywords Breast cancer - Skin-sparing mastectomy · Latissimus dorsi flap · Complications - Local recurrence . Metastases $\cdot$ Cosmetic

\section{Introduction}

Immediate breast reconstruction (IBR) is performed to improve body image and the remaining quality of life for women facing mastectomy [1-4]. Success can be defined as the best possible cosmetic result that does not sacrifice oncological safety [5, 6]. In recent years, skin-sparing mastectomy (SSM) in combination with IBR has gained in acceptance and popularity for early invasive or in situ disease (DCIS) in terms of both oncological safety and acceptable rates of postoperative complications [7-15]. More recent reports have also documented the effectiveness of SSM plus IBR for locally advanced disease [1624]. These studies showed complication rates comparable to those of a similar group of patients who did not undergo IBR. In addition, these studies found no significant differences in local relapse or distant metastasis rates for patients receiving IBR for locally advanced disease in comparison with patients without immediate reconstruction 
$[5,17,18,21,23]$. The risk of recurrence thus does not appear to be technique-dependent but instead is stagedependent, as tumor size and nodal involvement are significant risk factors [25, 26]. Last, overall survival and disease-free survival may not be affected by IBR for T1T3 tumors in comparison with mastectomy alone [27].

Adjuvant therapy is another concern regarding IBR. The international guidelines have extended the indications for chemotherapy (CT) to include early breast cancers in addition to locally advanced cancers. CT is indicated for both axillary-positive and axillary-negative patients on the basis of high-risk tumor status (diameter, SBR grading, negative hormone receptor status) [28]. It is now acknowledged that when SSM plus IBR is the primary treatment, it does not delay the start of CT [29-31]. On the other hand, randomized studies have demonstrated the usefulness of postmastectomy radiotherapy (PMRT) to decrease local recurrence and increase survival for patients with positive axillary lymph nodes $[32,33]$. This situation has increased the complexity of planning for IBR for invasive cancers. First, the need for radiation therapy (RT) cannot always be definitively established before surgery [34]. Second, PMRT is known to increase the surgical complication rate and to decrease the esthetic result of implant-based SSM [34]. Third, some studies have raised the concern that irradiation of the immediately reconstructed breast results in a lower quality of radiation. These studies showed that the majority of radiation plans are unsatisfactory in terms of providing broad coverage of the chest wall and internal mammary nodes while adequately sparing heart and lung [35-37]. Fourth, recent studies reported increased tumor recurrence and patient demise when RT was performed following breast reconstruction in comparison with RT delivered before reconstruction [37-39].

In order to safely integrate RT into the reconstructive algorithm, we have developed the concept of post-neoadjuvant therapy SSM plus IBR over the past 10 years. Essentially, mastectomy and immediate reconstruction are performed once CT and RT have been completed. We believe this concept has some clear advantages. First, SSM plus IBR can be offered to women with locally advanced cancers. Second, since surgery is the final step in the anticancer therapy, it does not interfere with either CT or RT. This study was thus performed to evaluate the feasibility, oncological safety, and esthetic outcome offered by this concept. Patients with invasive breast cancer who had undergone CT and RT followed by SSM plus latissimus dorsi (LD) flap-based IBR were compared with patients who had undergone modified radical mastectomy, adjuvant CT and RT, and finally LD flap-based delayed breast reconstruction during the same time period.

\section{Patients and methods}

Since January 2000, 648 consecutive patients underwent mastectomy for malignant disease in the Department of Gynecological Surgery of Arnaud de Villeneuve Teaching Hospital, University of Montpellier, France. All breast cancer patients were routinely managed by primary lumpectomy plus axillary dissection or, in cases of multifocality, core needle biopsy plus axillary dissection. All were evaluated by a multidisciplinary breast cancer team. The need for mastectomy was indicated by conservative treatment failure, discrepancy of tumor size and breast volume, multicentric disease, or invasive tumor associated with extensive DCIS. CT was decided on in accordance with current guidelines regarding patient age, radiologic tumor diameter, SBR grading, and estrogen receptor, Her-2-neu overexpression and axillary node status. RT was decided on in accordance with current guidelines regarding radiologic tumor diameter, multicentric disease, lymphovascular invasion, and axillary node status. Patients with non-metastatic invasive breast cancer and needing mastectomy, radiation therapy, and chemotherapy were candidates to enter the study. One hundred and sixty-four patients who met the inclusion criteria and who were candidates for breast reconstruction were offered the choice between the two oncological managements. Sixty patients were excluded from this study for various reasons (Fig. 1). Twentysix selected patients with stages IIA-IIIA breast cancer who wanted immediate breast reconstruction were offered post-neoadjuvant therapy SSM plus IBR. These patients received primary $\mathrm{CT}$ followed by preoperative $\mathrm{RT}$ and final-step SSM plus IBR with an LD flap (IBR group). The 78 patients who did not choose IBR underwent primary mastectomy followed by adjuvant CT and RT and finally LD delayed breast reconstruction (DBR group). The records were analyzed for patient characteristics, tumor patterns, treatment details, and oncological and cosmetic outcomes.

Systemic therapy varied over time and followed the conclusions in the published literature. Between 2000 and 2004, neoadjuvant (IBR group) and adjuvant (DBR group) CT consisted on FEC 100 [5-fluorouracil $500 \mathrm{mg} / \mathrm{m}^{2}$ intravenously (i.v.), epirubicin $100 \mathrm{mg} / \mathrm{m}^{2}$ i.v., and cyclophosphamide $500 \mathrm{mg} / \mathrm{m}^{2}$ i.v.] every 21 days for six cycles. As of 2005, neoadjuvant CT (IBR group) consisted on FEC 100 every 21 days for four cycles followed by paclitaxelbased CT $\left(175 \mathrm{mg} / \mathrm{m}^{2}\right.$ every 21 days) for four cycles. During the same period, adjuvant CT (DBR group) consisted on FEC 100 every 21 days for three cycles followed by paclitaxel-based CT $\left(175 \mathrm{mg} / \mathrm{m}^{2}\right.$ every 21 days $)$ for three cycles. In addition, after 2004, patients with Her-2neu overexpression ( $3+$ staining by fluorescent in situ hybridization or gene amplification in FISH) received 


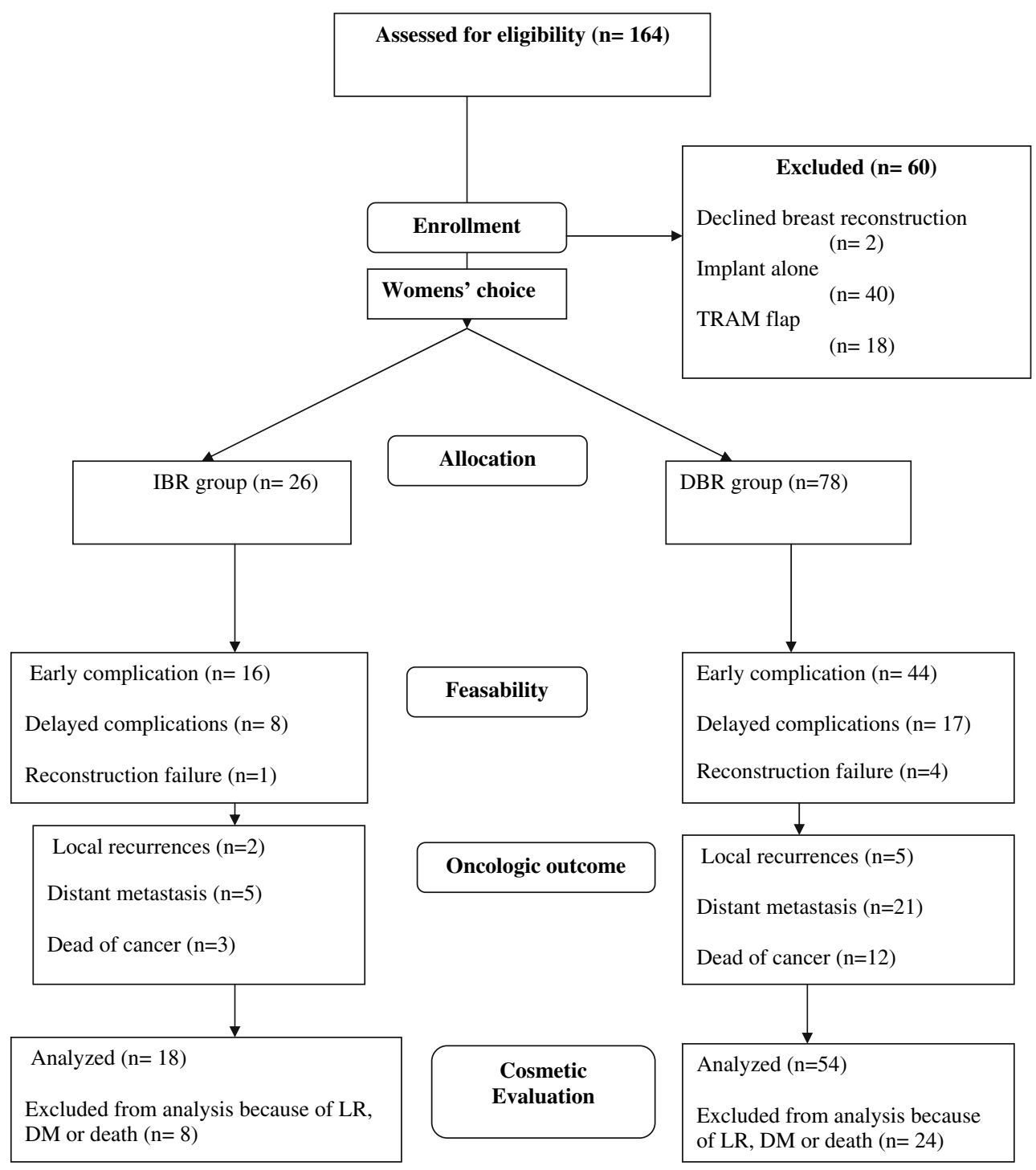

Fig. 1 Study flow chart

trastuzumab for 12 months. Patients who had hormone receptor-positive tumors also received tamoxifen or aromatase inhibitors for 5 years.

For the IBR patients, RT was delivered to the whole breast and the entire thickness of the chest wall by two opposed tangential fields (each field being treated every day) using photons (6MV) to a total dose of 50 Gy in 2-Gy daily fractions 5 days a week. For the DBR patients, RT was delivered to the entire thickness of the chest wall by en-face electrons (9MV) or by two tangential photon (6MV) fields to a total dose of 50 Gy in 2-Gy daily fractions 5 days a week. For both groups radiotherapy treatment was delivered in the supine position to ensure reproducibility during simulation and treatment. The planning target volume included the whole breast and/or the entire thickness of the chest wall and regional lymph nodes (i.e., supraclavicular and infraclavicular nodes, and the internal mammary nodes in the four upper intercostal spaces). The fields were defined on the CT-simulator or, after planning CT, conventional simulator. All shielding blocks were indicated on the digitally reconstructed radiographs or the simulation films, respectively. The field arrangement included the use of an anterior photon field in the supraclavicular region to a total dose of 44-50 Gy, and a mixture of anterior electrons and photons to the internal mammary nodes to a total dose of 44-50 Gy. Each field was calculated wit three-dimensional dosimetry.

In all cases, breast reconstruction was performed by the senior author (PLG) using a standardized LD flap procedure. A transverse skin island was usually designed over 
the upper portion of the muscle. With the patient in a lateral position, the dissection proceeded in the muscular plane in a caudal direction to the iliac crest and cranially to the scapular bone. The humeral attachment was divided when necessary to obtain adequate excursion. The thoracodorsal nerve was systematically preserved. The flap was passed under the axillary tunnel to the breast region and the patient was turned to the supine position to perform implant positioning and flap shaping. The implant was always placed above the pectoralis major muscle and underneath the LD flap. The LD flap was secured medially to the sternum, superiorly to the pectoralis muscle fascia, inferiorly to the inframammary crease, and laterally to the serratus anterior muscle to avoid lateral displacement of the implant. A suction drain was placed under the flap for 48 $72 \mathrm{~h}$, during which time the patient was given prophylactic intravenous antibiotics. The donor site was closed in two layers over the suction drain. Drainage was usually discontinued after three to 5 days or when the output was less than $50 \mathrm{cc}$ within a $24-\mathrm{h}$ period. The patient was then seen 1 week after discharge to review her wounds, after 3 months to assess the need for further surgery, and then every 6 months. Surgery for IBR patients was planned 8 10 weeks after the completion of RT. The mastectomy incision included an ellipse of skin with the nipple-areola excision and the inframammary fold (Fig. 2). The LD flap was harvested through a large transversal elliptical skin incision as needed to exactly match the mastectomy defect (Fig. 3). Surgery for DBR patients was scheduled six to 12 months weeks after RT. The LD flap was harvested through a transversal skin incision and positioned on the anterior chest wall, regardless of the previous mastectomy scar, to place the flap along the lateral, and inferior margins of the reconstructed breast. LD reconstruction was performed using permanent implants (McGhan style 410, McGhan style MLP, Allergan Medical, Marlow, UK) or

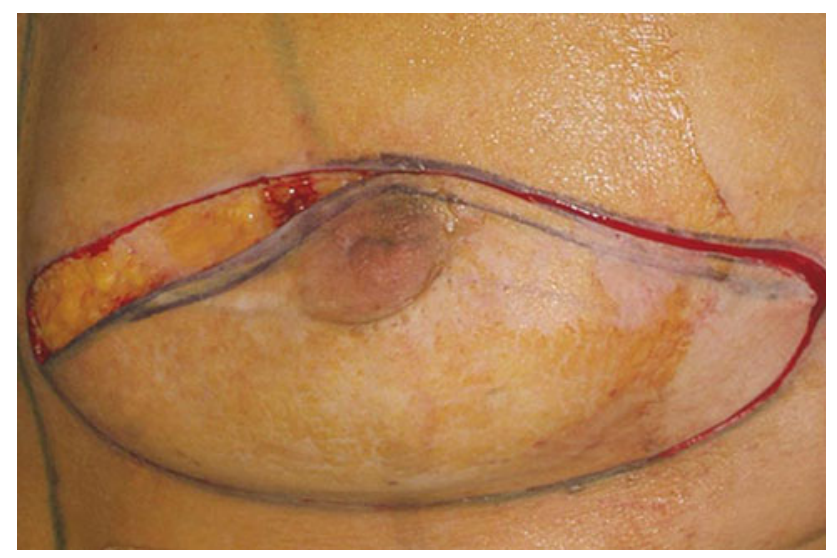

Fig. 2 IBR: design of the mastectomy incision expanders (McGhan 133 FV, Allergan Medical, Marlow, UK). For all patients in whom expanders were used at the first operation, surgical revision with a change to a definitive silicone implant was performed. During this step, capsulotomy and surgical refinements were performed as needed to obtain the best symmetry between the two breasts.

Tumor response to neoadjuvant therapy was evaluated in the IBR patients, who had no lumpectomy before chemotherapy. The two endpoints were clinical response (determined by physical examination) and pathologic response (determined by histologic examination). Clinical tumor size was ascertained before the initiation of $\mathrm{CT}$ and before surgery. When the disease was multifocal, the products of the two greatest perpendicular diameters of the tumor or tumors were calculated, and the sum of these measurements was recorded as "total tumor size." Clinical tumor response was defined as complete (cCR) if there was no clinical evidence of palpable tumor at the time of surgery [40]. A reduction in total tumor size of $50 \%$ or greater at the time of surgery was considered to be a clinical partial response (cPR). When there was an increase in total tumor size of more than $50 \%$ (compared with pretreatment measurements), the patient was considered to have progressive disease. Mastectomy specimens were analyzed to evaluate the pathologic tumor response. In accordance with recent expert recommendations, patients who had no invasive or in situ ductal cancer in the breast were considered to have had a pathologic complete response (pCR) [41]. In addition to pathologic response to preoperative treatment, the histologic patterns of the mastectomy specimens of IBR patients treated by primary lumpectomy are reported regarding residual in situ invasive carcinoma.

Postoperative outcomes were evaluated by two independent physicians (GR, PB). Follow-up was scheduled at intervals ranging from every 3 months to annually, depending on the length of time since treatment. All follow-up, whether performed by the radiation oncologist, medical oncologist, or surgeon, included physical examination of the breasts and regional lymph nodes. Follow-up also included CT scanning, breast MRI, contralateral mammogram if applicable, and serum tumor markers, at the discretion of the treating physicians. Recurrences and other treatment failures were documented by clinical examination, radiologic test, and/or pathologic assessment. Complications were classified as immediate when occurring within the first 30 days of breast reconstruction. Immediate complications were breast skin envelope necrosis (IBR group) and anterior thoracic wall skin necrosis (DBR group), marginal flap necrosis requiring wound care, hematoma requiring surgical revision, and implant infection requiring surgical revision. Dorsal seroma was defined by the presence of fluid collection at the 
Fig. 3 Early results in IBR patients. The volume of the reconstructed breast may have differed from that of the contralateral breast $(\mathbf{a}, \mathbf{b})$ to anticipate the symmetrization procedure or to follow the patient's wishes
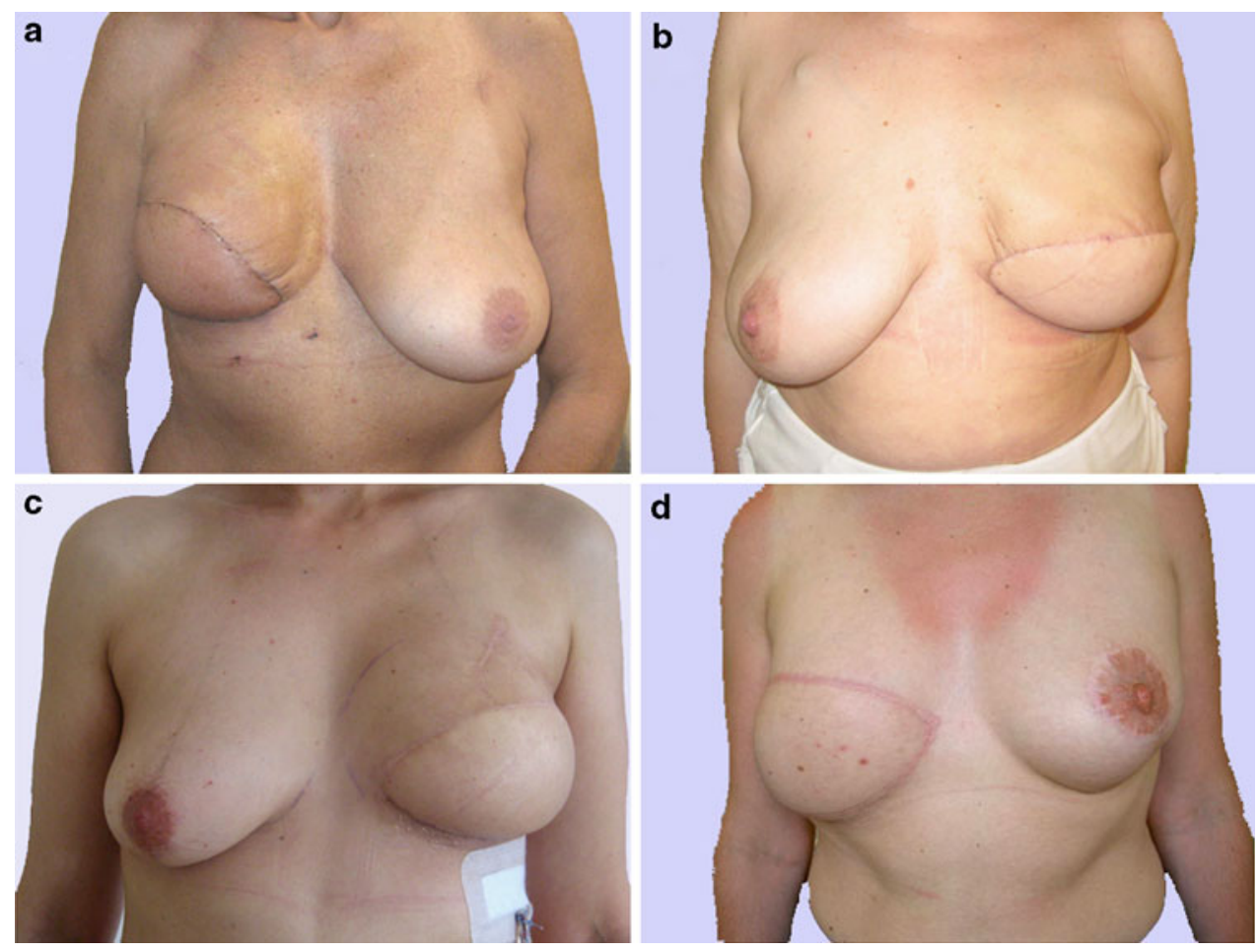

dorsal site after the drain was removed, requiring one or more needle evacuations to relieve the symptoms. Back skin flap necrosis was defined by skin necrosis needing wound care (Fig. 4). The early implant loss rate was recorded. Delayed complications were capsular contracture, back pain, and reconstruction failure. Implant revision was differentiated to distinguish between revision for capsular contracture and for an esthetic indication, which was not recorded as a late complication. The degree of capsular contracture was assessed by the surgeon during patient follow-up using the Baker classification. Breast reconstructions with Baker classes 3 and 4 were classified as capsular contracture complications. Reconstruction failure was defined as breast reconstruction being abandoned or achieved by another surgical procedure. Local recurrence (LR) was defined as histologically proven recurrent tumor in either the ipsilateral breast skin or the chest wall after the mastectomy. Tumor spread to the internal mammary, supraclavicular, infraclavicular, or ipsilateral axillary nodes, or to the nonbreast skin of the ipsilateral chest wall, was classified as other locoregional recurrence. All other sites of tumor recurrence were classified as distant metastases.

The postoperative esthetic results were evaluated by two independent physicians (GR, PB), who reviewed the photographs or saw the patients themselves at the last followup visit. Patients who were not disease-free at the visit were not evaluated for cosmetic result. The esthetic result was stratified by subscales according to Gerber et al. [24].

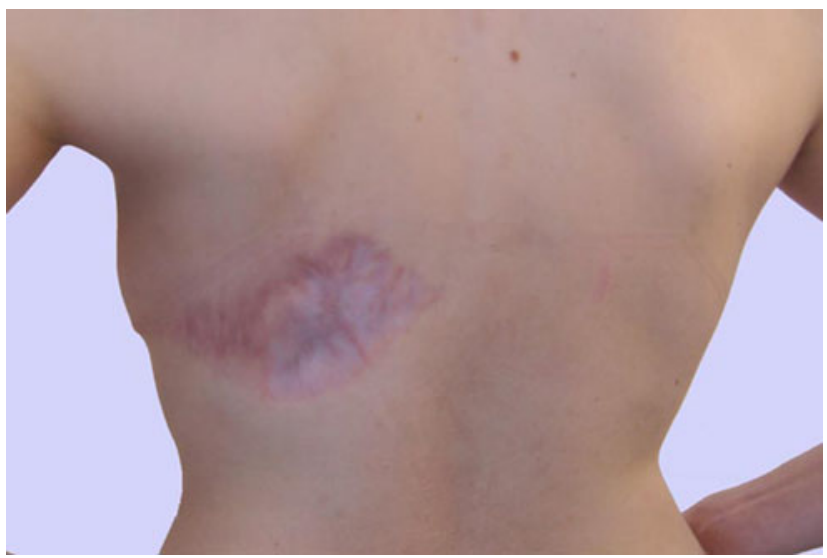

Fig. 4 Aspect of the dorsal scar on an IBR patient after back skin necrosis

Briefly, the volume of the reconstructed breast, shape of the breast mounds, symmetry, ipsilateral and contralateral scars, and the inframammary fold were each evaluated from 0 to 2 points. The results were defined as follows: excellent (Fig. 5): 10-8 points; good (Fig. 6): 7-6 points; fair (Fig. 7): 5-4 points; or poor: fewer than 4 points. In addition, patient satisfaction was assessed by subjective questionnaire using the same grading scale.

Statis tical analysis of the data was performed with StatView software (StatView 512, Brain Power, Inc., Calabasas, CA). The data analyst was blinded to the surgery groups. First, descriptive statistics were performed for the variables. The computed statistics included mean 
Fig. 5 Cosmetic Results evaluated as excellent. a One year post-reconstruction in a 42 year-old patient, and 3 months post-symmetrization and nipple reconstruction. b Five years post-reconstruction in a 63 year-old patient

Fig. 6 Cosmetic results evaluated as good. a One year post-reconstruction in a 55year-old patient, and 2 months post-symmetrization and nipple reconstruction. b Five years post-reconstruction in a 48 year-old patient
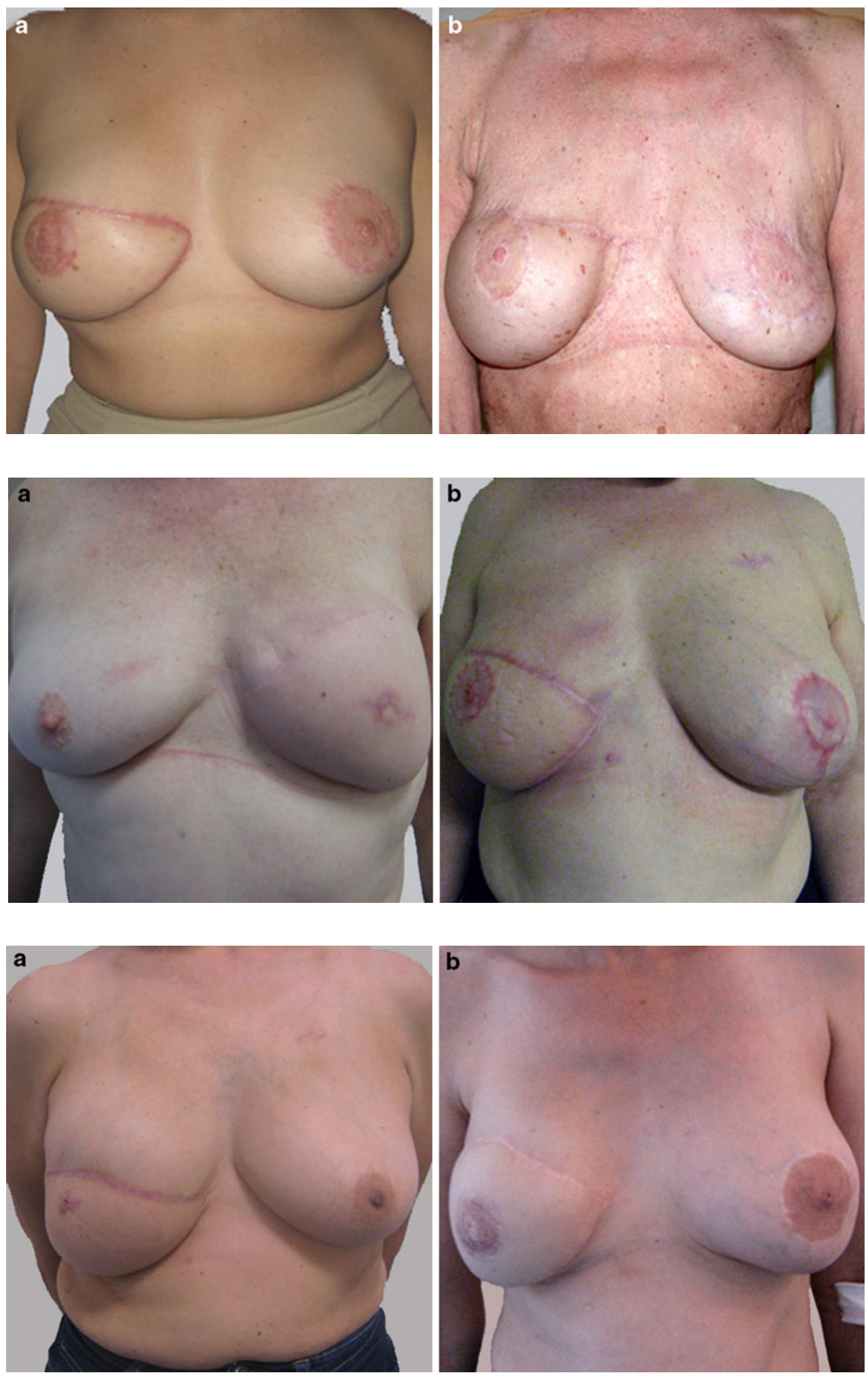

Fig. 7 Cosmetic results evaluated as fair. a One year post-reconstruction in a 56year-old patient, and 3 months post-symmetrization and nipple reconstruction. b Five years post-reconstruction in a 50year-old patient and standard deviation (SD) of continuous variables, frequencies, and relative frequencies of categorical factors. Baseline preoperative variables were compared using the Chi-square test for categorical data or, when appropriate, Fisher's exact test. The Mann-Whitney $U$ test was used to compare the medians of non parametric variables. $P$ value $<0.05$ was considered to be statistically significant. 


\section{Results}

Oncological results

The characteristics of the IBR and DBR groups are detailed in Tables 1 and 2. The two groups were comparable in terms of body mass index, percentages of smokers and diabetic patients, and duration of follow-up. However, the patients who chose IBR were significantly younger than the DBR patients $(P=0.006)$. As expected, the mean (SD) interval between completion of RT and surgery was 8.5 weeks (1.06) in the IBR group versus 29.1 weeks (5.7) in the DBR group $(P<0.0001)$.

Of the 26 breast carcinomas treated in the IBR group, six were lobular carcinomas and 20 were ductal carcinomas. Fourteen were managed by primary lumpectomy plus axillary dissection before initiation of chemotherapy. The remaining 12 were staged by core needle biopsy plus axillary dissection before initiation of CT and were assessable for clinical and pathologic response after neoajuvant therapy. The pre therapeutic histologic characteristics and the post-neoadjuvant therapy changes are reported on Table 3 . Clinical complete response was observed in five of the $12(41 \%)$ assessable patients. The overall clinical response rate (cCR $+\mathrm{cPR}$ ) was $58 \%$ (7 of 12 patients). No patient had clinically progressive disease. Two patients had no histologic evidence of cancer in the breast ( $\mathrm{pCR}=16.6 \%$ ). For IBR patients who were managed by primary lumpectomy plus axillary dissection, the response to neoadjuvant therapy was not available. However, invasive residual disease was found in two patients and residual DCIS in five patients.

Initial surgery in both the IBR and DBR groups consisted of LD flap plus expanders in five (19.3\%) and nine $(11.5 \%)$ cases, respectively, and silicone implants in 21 $(80.7 \%)$ and $69(88.5 \%)$ cases, respectively $(P=0.482)$. To obtain a final symmetry that was acceptable to both patient and physician, a contralateral breast operation including reduction or augmentation mammaplasty was performed in seven (26.9\%) patients of the IBR group
Table 1 Characteristics of the 93 women in the study

Data are presented as mean (SD) or $n(\%)$

* Mann-Whitney $U$ test

** Chi-square test

Table 2 Details of histologic findings

Data are presented as $n(\%)$

* Chi-square test

a American Joint Committee on Cancer (AJCC) stage groupings for breast cancer using the T, N, M translated to stage 0 , I, II, III \& IV

\begin{tabular}{llll}
\hline & IBR group $N=26$ & DBR group $N=78$ & $P$ \\
\hline Age at time of surgery (years) & $50.6(10.4)$ & $56.2(9.3)$ & $0.006^{*}$ \\
Body mass index $\left(\mathrm{kg} / \mathrm{m}^{2}\right)$ & $24.3(1.62)$ & $24.6(2.5)$ & $0.357^{*}$ \\
Length of follow-up (years) & $4.7(1.91)$ & $4.1(2.2)$ & $0.132^{*}$ \\
Smokers & $3(11.5)$ & $10(12.8)$ & $0.863^{* *}$ \\
Diabetes mellitus & $1(3.4)$ & $4(5.1)$ & $0.786^{* *}$ \\
\hline
\end{tabular}

\begin{tabular}{|c|c|c|c|}
\hline & IBR group $N=26$ & DBR group $N=78$ & $P$ \\
\hline AJCC staging $^{a}$ & & & $0.235^{*}$ \\
\hline Stage IIA/IIB & $24(92.2)$ & $70(90)$ & \\
\hline Stage IIIA & $2(7.8)$ & $8(10)$ & \\
\hline Axillary involvement & & & $0.409 *$ \\
\hline Negative & 0 & 2 & \\
\hline Positive & 26 & 76 & \\
\hline SBR grading & & & $0.545^{*}$ \\
\hline 1 & $1(3.8)$ & $6(8.9)$ & \\
\hline 2 & $16(61.5)$ & $39(50)$ & \\
\hline 3 & $9(34.7)$ & $33(42.3)$ & \\
\hline Multifocality/multicentricity & $12(46.1)$ & $44(56.4)$ & $0.363 *$ \\
\hline Extensive DCIS & $9(34.6)$ & $19(24.3)$ & $0.315^{*}$ \\
\hline Lymphovascular invasion & $4(15.3)$ & $15(19.2)$ & $0.655^{*}$ \\
\hline Estrogen receptors & & & $0.909 *$ \\
\hline Positive & $15(57.6)$ & $34(43.5)$ & \\
\hline Negative & $11(42.4)$ & $44(56.5)$ & \\
\hline Her-2-neu & & & $0.468 *$ \\
\hline Positive & $7(27)$ & $22(28.2)$ & \\
\hline Negative & $13(50)$ & $46(58.9)$ & \\
\hline Unknown & $6(23)$ & $10(12.8)$ & \\
\hline
\end{tabular}


Table 3 Distribution of histological findings before chemotherapy and after neoadjuvant therapy for IBR patients

\begin{tabular}{lll}
\hline & $\begin{array}{l}\text { Lumpectomy }+\mathrm{AD} \\
N=14\end{array}$ & $\begin{array}{l}\text { Biopsy }+\mathrm{AD} \\
N=12\end{array}$ \\
\hline Pre therapeutic & & \\
Lobular carcinoma & 2 & 4 \\
Ductal carcinoma & 12 & 8 \\
Multifocality & 3 & 9 \\
DCIS & 7 & 2 \\
Post therapeutic & & \\
cCR & $\mathrm{ND}$ & 5 \\
cPR & $\mathrm{ND}$ & 2 \\
pCR & $\mathrm{ND}$ & 2 \\
Residual DCIS & 5 & \\
Residual invasive cancer & 2 &
\end{tabular}

Data are presented as $n$

$A D$ axillary dissection, $c C R$ clinical complete response, $c P R$ clinical partial response, $p C R$ pathologic complete response

versus $26(33.3 \%)$ of the DBR group $(P=0.715)$. Last, nipple-areola reconstruction was done in $21(80.7 \%)$ IBR patients versus $56(71.7 \%)$ in the DBR group $(P=0.081)$.

\section{Complications}

Sixteen $(61 \%)$ early complications were reported for the IBR group versus $44(56 \%)$ for the DBR group $(P=$ $0.645)$. No differences were found between the two groups for the rate of breast skin envelope necrosis, marginal LD flap necrosis, implant infection, hematoma, or dorsal seroma. Conversely, the rate of marginal back skin flap necrosis was higher in the IBR group than in the DBR group (Table 4). Breast skin envelope necrosis (IBR group), anterior thoracic wall necrosis (DBR group), marginal LD flap necrosis, and back skin flap necrosis were managed routinely by wound dressing. Implant infection

Table 4 Distribution of observed immediate complications

\begin{tabular}{llll}
\hline & $\begin{array}{l}\text { IBR group } \\
N=26\end{array}$ & $\begin{array}{l}\text { DBR group } \\
N=78\end{array}$ & $P$ \\
\hline Breast skin envelope necrosis* & $1(3.8)$ & $4(5.1)$ & $0.786^{* *}$ \\
Marginal LD flap necrosis & $2(7.6)$ & $7(8.9)$ & $0.708^{* *}$ \\
Hematoma & $3(11.5)$ & $10(12.8)$ & $0.863^{* *}$ \\
Implant infection & $1(3.8)$ & $4(5.12)$ & $0.786^{* *}$ \\
Dorsal seroma & $4(15.3)$ & $14(17.9)$ & $0.762^{* *}$ \\
Marginal back skin flap necrosis & $5(19.3)$ & $4(5.1)$ & $0.04^{* *}$ \\
Total early complications & $16(61.5)$ & $44(56.4)$ & $0.645^{* *}$ \\
\hline
\end{tabular}

Data are presented as $n(\%)$

* Breast skin envelope necrosis (IBR group) and anterior thoracic wall skin necrosis (DBR group)

** Chi-square test required surgical revision, and an attempt to conserve the implant was made using irrigation and drainage. In the IBR group, all infected implants were managed conservatively. In the DBR group, one of the four infected implants was removed after failure of conservative management. The early implant loss rate was $0 \%$ in the IBR group versus $12 \%$ in the DBR group. Eight late complications were reported for the IBR group (30\%) versus 17 (21\%) for the DBR group $(P=0.362)$. The rate of capsular contracture (CC) was not different between groups. The total number of implant revisions, including expander change for definitive implant, surgical cure of $\mathrm{CC}$, and revision for esthetic reasons, was not different in the IBR group in comparison with the DBR group. The rate of reconstruction failure was the same in the two groups. Finally, the number of surgical procedures to achieve symmetrization was the same in the two groups (Table 5).

\section{Follow-up}

The mean (SD) follow-up was 4.7 years (1.9) in the IBR group and 4.5 years $(1.8)$ in the DBR group $(P=0.751)$. No patients were lost to follow-up. The overall LR rates amounted to $7.7 \%$ ( 2 out of 26 patients) in the IBR group and $6.4 \%$ (5 out of 78 patients) in the DBR group $(P=0.823)$. For the IBR and DBR patients, four $(57 \%)$ of the seven LRs were detected clinically and the three others were detected by thoracic tomodensitometry performed because of axillary venous thrombosis ( 2 cases) and arm lymphedema ( 1 case). Treatment of LR in the IBR group consisted of local excision for one case and local excision plus CT for the other case. In the DBR group, LR treatment consisted of local excision alone in two cases and excision plus CT in three cases. Five patients (19.2\%) of the IBR group developed distant metastasis versus 21 (26.9\%) in the DBR group $(P=0.602)$. During the study period, five patients $(19.2 \%)$ in the IBR group died of cancer disease versus nine $(13.5 \%)$ in the DBR group $(P=0.482)$.

Table 5 Distribution of delayed complications

\begin{tabular}{llll}
\hline & $\begin{array}{l}\text { IBR group } \\
N=26\end{array}$ & $\begin{array}{l}\text { DBR group } \\
N=78\end{array}$ & $P$ \\
\hline Capsular contracture & $4(15.3)$ & $9(11.5)$ & $0.616^{*}$ \\
Back pain & $3(11.5)$ & $4(8.9)$ & $0.786^{*}$ \\
Reconstruction failure & $1(3.8)$ & $4(5.1)$ & $0.786^{*}$ \\
Total late complications & $8(30.7)$ & $17(21.7)$ & $0.362^{*}$ \\
Implant revision capsule & $4(15.3)$ & $9(11.5)$ & $0.641^{*}$ \\
Total implant revision & $9(34.6)$ & $18(28.1)$ & $0.245^{*}$ \\
Symmetrization procedure & $7(26.9)$ & $26(33.3)$ & $0.715^{*}$ \\
\hline Data are presented as $n(\%)$ & & & \\
$*$ Chi-square test & & &
\end{tabular}


At the last follow-up visit, esthetic outcome was evaluated for disease-free patients and patients with no reconstruction failure. Eighteen IBR patients were evaluated versus 54 DBR patients. At this last visit, $77.7 \%$ of the IBR patients (14 out of 18) had an overall esthetic result of excellent or good versus $87 \%$ (47 out of 54) for the DBR patients. The evaluation by the patients themselves was identical in the two groups (Table 6).

\section{Discussion}

This study shows that post-neoadjuvant therapy SSM plus IBR offers the same feasibility, oncological safety, and cosmetic results as DBR.

The IBR patients were younger than the DBR patients. Although this may have introduced bias in the comparison of safety, other important factors of co-morbidity (BMI, diabetes mellitus, smoking) were comparable between the two groups. According to Lipa et al. [42], age becomes a significant risk factor for perioperative complications in women over 65 years in comparison to younger women. In our study, the mean age of the DBR group was 56 years and, like other authors $[42,43]$, we believe that the general health status of patients is a better indicator than crude age. For Pinsolle et al. [43], the only factor found to be significantly associated with anterior thoracic wall skin necrosis and flap necrosis was cigarette smoking. This condition was equally distributed between our two groups. Age over 50 years and BMI higher than 25 were not associated with an increased rate of complications. Regarding the hematoma rate, Pinsolle et al. [43] showed that BMI over 25 was a significant risk factor, as was the postoperative use of low-molecular-weight heparin. In our study, these conditions did not differ between the IBR and DBR patients. Last, obesity is another proven risk factor for postoperative infection [42, 44] but was equally distributed in our two groups of patients.

Early complications were mainly benign in both groups. A slight but significant difference was seen regarding back skin necrosis. We believe this difference was not due to the timing of RT, but rather to the surgical technique. Indeed,

Table 6 Cosmetic evaluation

\begin{tabular}{llllllll}
\hline & \multicolumn{3}{l}{ Medical evaluation } & & \multicolumn{3}{l}{ Patient evaluation } \\
\cline { 2 - 3 } & IBR & DBR & $P$ & IBR & DBR & $P$ \\
\hline Excellent & $5(27.7)$ & $21(38.8)$ & $0.541^{*}$ & $9(50)$ & $29(53.7)$ & $0.723^{*}$ \\
Good & $9(50)$ & $26(48.2)$ & & $7(38.8)$ & $22(40.7)$ & \\
Fair & $4(22.3)$ & $7(13)$ & & $2(11.2)$ & $3(5.6)$ & \\
\hline
\end{tabular}

Data are presented as $n(\%)$

* Chi-square test although the size of the skin island was not recorded at surgery, we assume that the size of the dorsal skin island was larger in the IBR group than in the DBR group. In the IBR group, a large dorsal skin flap was undermined to match the large ellipse of breast skin and the inframammary fold that had been removed. Conversely, the dorsal skin flap was smaller in the DBR group because large skin coverage was unnecessary. Interestingly, complications related to post-radiation skin damage, such as skin necrosis or implant infection, were not over-reported in the IBR group in comparison with the DBR group, indicating that the short time between RT and SSM in the IBR group may not have increased the deleterious effects of radiation. Comparison with the literature was limited by the few reports concerning IBR after CT and RT. However, our concept carries complication rates comparable to those of other published series about implant reconstruction after radiation therapy. An interesting paper by Michy et al. [45] reported a series of 101 patients who had undergone IBR after neoadjuvant $\mathrm{CT}$ and RT for invasive breast carcinoma. IBR was accomplished by transverse rectus abdominis musculocutaneous flap (TRAM) in 38 patients, by latissimus dorsi musculocutaneous flap with prosthesis (LDMP) in 32, by autologous latissimus dorsi musculocutaneous flap (ALDM) in 15, and by simple prosthetic implant in 26. The respective complication rates were 50, $37.5,8$, and $62 \%$. The need for additional surgical procedures was, respectively, 29, 15, 6, and 56\% [45]. A recent series reported by Tallet et al. [37] concerned 77 patients undergoing IBR with tissue expanders and implants. Of the 55 patients who had received RT, 51\% developed postoperative complications versus $14 \%$ for the non-irradiated patients. Interestingly, a small sample of eight patients had been irradiated before reconstruction because of ipsilateral recurrence. The complication rate was comparable in this sample, with five of the eight patients presenting a complication [37]. Spear and Onyewu recently found that, of 40 patients treated from 1990 to 1998 who underwent staged breast reconstruction with an expander and implant and received radiation before, during or after the expansion, $47.5 \%$ required an additional flap procedure to improve, correct, or save the implant [46]. The overall complication rate was $52.5 \%$, compared with $10 \%$ in a control group of 40 patients who did not undergo irradiation who were randomly sampled from 200 such patients treated during the same period. Based on the data from retrospective series of SSM plus IBR, the complication rates range from 14 to $55 \%$ when RT is administered versus 7 to $31 \%$ for non-irradiated patients [38]. However, the complication rates seem to be comparable when RT is delivered before and after breast reconstruction [37, 38].

Capsular contracture is a common mid- to long-term complication associated with implant-assisted breast 
reconstruction plus $\mathrm{RT}$ and a major concern for the esthetic result. We examined the difference in the rate of $\mathrm{CC}$ between our concept (post-neoadjuvant IBR) and other surgical strategies evaluated directly in this comparative study or reported in the literature. Our results demonstrated that the CC rate was similar in the IBR and DBR groups (15.3 and $11.9 \%, P=0.616$ ), as was the esthetic outcome, which is known to be highly dependent on the CC rate [47]. Furthermore, the capsular formation rate compared favorably with the rates reported in series of immediate breast reconstructions plus adjuvant RT [37, 46-48]. The CC rate varies considerably in the literature-from 17 to $68 \%-$ because of the inhomogeneity of the selected patients and the surgical procedures used. The reports in fact concern not only one- or two-stage implant-based reconstructions, but also latissimus flaps plus implants. Another major concern in the validation of our concept was the influence of RT timing on capsular formation. Few data have been published and the debate is not yet closed. Behranwala et al. [49] reported a study of 136 breast reconstructions in 114 patients. A total of 44 patients underwent RT, 15 before breast reconstruction and 29 after reconstruction. The risk of capsule formation was lower for the patients irradiated before reconstruction in comparison with PMRT [HR: $2.23(0.61-8.2)$ versus $5.23(2.31-11.8), P<0.001$ ] [49]. This seems logical because the flap used for the reconstruction, as well as the implant, was unexposed to irradiation. Pinsolle et al. also evaluated the CC rate in relation to the timing of RT in a series of 266 IBR and reported similar results. For patients irradiated before IBR, the CC rate was $24 \%$ in comparison with $55 \%$ when RT was performed after IBR [43]. Conversely, Contant et al. [50] examined the medical records of 100 women who had undergone a mastectomy followed by IBR with a subpectorally placed silicone prosthesis. Thirteen prostheses were implanted prior to RT and 15 prostheses were implanted after irradiation of the chest wall. The risk of capsule formation did not differ for patients irradiated before reconstruction in comparison with PMRT [HR: 7.5 (3.416.6) vs. 6.5 (2.4-15.8)] [50]. However, this series was too small to offer sufficient statistical power. Last, our CC rate seems to be comparable to the reported rates after IBR using LD flaps for salvage mastectomy following conservative treatment failure (surgery plus RT). In a series of 57 patients undergoing salvage mastectomy plus LD-based IBR, Disa et al. [51] reported a CC rate of $17 \%$. Freeman et al. presented a series of 12 patients who underwent LD flap reconstruction after the development of recurrent cancer after breast conserving therapy. Despite previous radiation, the $\mathrm{CC}$ rate was $12.5 \%$ (median follow-up: 50 months; range: 20-93 months) and all 12 patients had a satisfactory esthetic result [52]. Moreover, the role of the LD flap with a prosthesis in reconstruction of the previously irradiated breast was examined in a retrospective review by Spear et al. [53]. Twenty-eight patients with available charts had undergone LD plus implant reconstruction. The average follow-up was 28.8 months (range: 1 week to 7 years). All patients had soft breasts at followup, with no evidence of capsular contracture [53]. The CC rates that we report in our series are closer to the rates for RMD and IBR for salvage mastectomy than for IBR and RT post-IBR. However, these results should be confirmed by direct comparison in much larger series.

The LR and cancer-related death rates were not significantly different in the two groups and compared favorably with the findings of other published series of patients who underwent SSM and patients with locally advanced disease who were treated by mastectomy with or without reconstruction $[5,54,55]$. The recurrence rates reported in the literature increased with stage, from $3 \%$ for stage I to $11.1 \%$ for stage III, from $3.7 \%$ for $\mathrm{T} 1$ to $9.9 \%$ for $\mathrm{T} 2-\mathrm{T} 4$, and from $0 \%$ for stage I to $25 \%$ for stage III $[9,11,13]$. Recurrence appeared to be stage-dependent and independent of the use of IBR. In addition, this wide range is possibly due to potential bias because of inadequate axillary sampling, incomplete surgical technique, and suboptimal systemic therapy [56]. A recent pooled analysis by Jatoi and Proschan of six randomized trials comparing mastectomy and breast conservation in the treatment of primary breast cancer examined mortality and LR [57]. The rates of LRR after mastectomy in these trials ranged from 2 to 16\% [57]. Slavin et al. [8] did not observe an increased risk of LR in a series of 51 IBRs with autologous tissue. Kroll concluded from the IBR series from M.D. Anderson that SSM did not increase the risk of LR [54]. Sandelin found an $8 \%$ LR rate in a series of 100 IBRs with a mean follow-up of 36 months [58]. Thus, the literature clearly demonstrates that the type of reconstruction has no influence on the clinical outcome of the disease, as was noted in our series. We conclude that our surgical procedure is a safe option, and after these preliminary results we found no difference in comparison with standard approaches using delayed breast reconstruction. However, our study findings would be strengthened by a longer followup, as LR rates and cancer-related death are known to increase over time $[20,55,59,60]$.

The combination of autogenous tissue/prosthetic reconstruction has several advantages in the setting of previously irradiated tissues. Autogenous tissue flaps provide pliable, well-vascularized soft tissue, which can facilitate both wound healing and the process of tissue expansion. The elective use of flaps alongside breast prostheses in patients irradiated before reconstruction is supported by many authors [61]. However, data on longterm esthetic outcomes are needed, particularly after RT, which is known to affect the esthetic results with time. 
Geber et al. reported the cosmetic results in a series of SSM patients after a mean follow-up of 59 months and, more recently, after a mean follow-up of 101 months [24, 62]. The surgeons' re-evaluation of the esthetic results revealed a significant shift from $78.4 \%$ excellent results after 59 months of follow-up to $47.9 \%$ after 101 months [24, 62]. Similarly, Clough et al. [47] reported deterioration in the esthetic outcome following implant-based IBR in a series of patients in whom only $8 \%$ had RT: from $86 \%$ acceptable results at 2 years to $54 \%$ at 5 years. Last, Thomson et al. [63] reported a prospective study of cosmetic outcome in immediate LD breast reconstruction with and without RT. The cosmetic outcome of all reconstructions deteriorated over time, with the non-irradiated reconstruction producing the best long-term esthetic outcome and the irradiated implant reconstructions faring worst. Adjuvant RT was the most important factor for the long-term decreased cosmetic score, although factors other than capsule formation, such as the natural aging of the contralateral breast parenchyma and the overlying skin envelope, as well as overall weight change, are likely to significantly influence the long-term score [63].

This study was limited by the possibility of selection bias and the short median follow-up. It would have been powerful to compare IBR group with a control group composed of patients treated with mastectomy and IBR followed by RT. However, in our practice, patients who have undergone mastectomy plus IBR for invasive disease have had false negative sentinel node status at introperative pathologic examination and had not received preoperative chemotherapy. In addition, in these cases, IBR was routinely performed using an implant alone or with expanders and not a LD flap. These conditions explain the lack of a control group. In the current study, the CT regimens used the same drugs in the two groups. However, since 2005, the total number of cycles administered has differed depending on whether CT is used as neoadjuvant therapy (IBR group, 8 cycles) or adjuvant therapy (DBR group, 6 cycles). This condition may have introduced a bias in the comparison of oncological outcome. A meta-analysis from Maury et al. [64] nevertheless showed that neoadjuvant and adjuvant $\mathrm{CT}$ had equivalent rates of survival and disease progression. Another limitation was the comparison of tumor response to treatment in each group. A close comparison of clinical and pathologic responses between groups was irrelevant because the DBR patients underwent mastectomy as the primary step and CT as adjuvant treatment. In addition, the number of patients assessable for tumor response to neoadjuvant treatment was small, as only 12 IBR patients had no lumpectomy before induction of CT. However, our study was not performed to assess and compare the responses to $\mathrm{CT}$ in the two groups. In the IBR group, neoadjuvant $\mathrm{CT}$ and RT were not performed to shrink tumor size and allow conservative treatment but to integrate IBR for patients needing both mastectomy and CT plus RT. An analysis of the LR rate by disease stage was not done because of the relatively low patient numbers. Although the majority of local failures occurred within the first 5 years of treatment, distant failure may occur later, and the patients in our study remain at risk for both locoregional and distant failure. Similarly, severe CC may not develop until several years after treatment [63] and the short median follow-up time of our study may give a falsely reassuring picture concerning our concept. As well, cosmesis outcome is known to decrease with time, particularly when RT is used. Thus, differences between the two groups in terms of LR and/or distant metastasis, CC rate and cosmesis outcome may appear at a later time. Despite these limitations, this report presents convincing evidence that our concept provides a basis for offering more women the opportunity to elect for immediate reconstruction, even in the setting of radiation therapy.

\section{Conclusion}

IBR is indubitably a valuable addition to the oncological surgical armamentarium for primary treatment of breast cancer. Our study shows that the feasibility and oncological safety of IBR are comparable to DBR. Our concept should be further analyzed in larger comparative studies in order to confirm our preliminary finding that IBR can be safely integrated into the global management of locally advanced breast cancer patients.

\section{References}

1. Dean C, Chetty U, Forrest APM (1983) Effects of immediate breast reconstruction on psychosocial morbidity after mastectomy. Lancet 1:459-462

2. Fernandez-Delgado J, Lopez-Pedraza MJ, Blasco JA et al (2008) Satisfaction with and psychological impact of immediate and deferred breast reconstruction. Ann Oncol 19:1430-1434

3. Rowland JH, Desmond KA, Meyerowitz BE et al (2000) Role of breast reconstructive surgery in physical and emotional outcomes among breast cancer survivors. J Natl Cancer Inst 92:1422-1429

4. Isern AE, Tengrup I, Loman $\mathrm{N}$ et al (2008) Aesthetic outcome, patient satisfaction, and health-related quality of life in women at high risk undergoing prophylactic mastectomy and immediate breast reconstruction. J Plast Reconstr Aesthet Surg 61:11771187

5. Downes KJ, Glatt BS, Kanchwala SK et al (2005) Skin-sparing mastectomy and immediate reconstruction is an acceptable treatment option for patients with high-risk breast carcinoma. Cancer 103:906-913

6. Bezuhly M, Temple C, Sigurdson LJ et al (2009) Immediate postmastectomy reconstruction is associated with improved breast cancer-specific survival: evidence and new challenges 
from the surveillance, epidemiology, and end results database. Cancer 115:4648-4654

7. Kroll SS, Schusterman MA, Tadjalli HE et al (1997) Risk of recurrence after treatment of early breast cancer with skin-sparing mastectomy. Ann Surg Oncol 4:193-197

8. Slavin SA, Schnitt SJ, Duda RB et al (1998) Skin-sparing mastectomy and immediate reconstruction: oncologic risks and aesthetic results in patients with early-stage breast cancer. Plast Reconstr Surg 102:49-62

9. Simmons RM, Fish SK, Gayle L et al (1999) Local and distant recurrence rates in skin-sparing mastectomies compared with non-skin-sparing mastectomies. Ann Surg Oncol 6:676-681

10. Spiegel AJ, Butler CE (2003) Recurrence following treatment of ductal carcinoma in situ with skin-sparing mastectomy and immediate breast reconstruction. Plast Reconstr Surg 111:706711

11. Carlson GW, Styblo TM, Lyles RH et al (2003) The use of skin sparing mastectomy in the treatment of breast cancer: the Emory experience. Surg Oncol 12:265-269

12. Mokbel K (2003) Towards optimal management of ductal carcinoma in situ of the breast. Eur J Surg Oncol 29:191-197

13. Medina-Franco H, Vasconez LO, Fix RJ et al (2002) Factors associated with local recurrence after skin-sparing mastectomy and immediate breast reconstruction for invasive breast cancer. Ann Surg 235:814-819

14. Rainsbury M (2006) Skin-sparing mastectomy. Br J Surg 93:276281

15. Patani N, Devalia H, Anderson A et al (2007) Oncological safety and patient satisfaction with skin-sparing mastectomy and immediate breast reconstruction. Surg Oncol 17:97-105

16. Godfrey PM, Godfrey NV, Romita MC (1995) Immediate autogenous breast reconstruction in clinically advanced disease. Plast Reconstr Surg 95:1039-1044

17. Styblo TM, Lewis MM, Carlson GW et al (1996) Immediate breast reconstruction for Stage III breast cancer using transverse rectus abdominis musculocutaneous (TRAM) flap. Ann Surg Oncol 3:375-380

18. Newman LA, Kuerer HM, Hunt KK et al (1999) Feasibility of immediate breast reconstruction for locally advanced breast cancer. Ann Surg Oncol 6:671-675

19. Foster RD, Esserman LJ, Anthony JP et al (2002) Skin-sparing mastectomy and immediate breast reconstruction: a prospective cohort study for the treatment of advanced stages of breast carcinoma. Ann Surg Oncol 9:462-466

20. Fersis N, Hoenig A, Relakis K et al (2004) Skin-sparing mastectomy and immediate breast reconstruction: incidence of recurrence in patients with invasive breast cancer. The Breast 13:488-493

21. Greenway RM, Schlossberg L, Dooley WC (2005) Fifteen-year series of skin sparing mastectomy for stage 0 to 2 breast cancer. Am J Surg 190:918-922

22. Meretoja TJ, von Smitten KA, Leidenius MH et al (2007) Local recurrence of stage 1 and 2 breast cancer after skin-sparing mastectomy and immediate breast reconstruction in a 15 -year series. Eur J Surg Oncol 33:1142-1145

23. Wright JL, Cordeiro PG, Ben-Porat L et al (2008) Mastectomy with immediate expander-implant reconstruction, adjuvant chemotherapy, and radiation for stage II-III breast cancer: treatment intervals and clinical outcomes. Int J Radiat Oncol Biol Phys 70:43-50

24. Gerber B, Krause A, Dieterich M et al (2009) The oncological safety of skin sparing mastectomy with conservation of the nipple-areola complex and autologous reconstruction: an extended follow-up study. Ann Surg 249:461-468

25. Carlson GW, Styblo TM, Lyles RH et al (2003) Local recurrence after skin-sparing mastectomy: tumor biology or surgical conservatism? Ann Surg Oncol 10:108-112
26. Carlson GW, Page A, Johnson E et al (2005) Local recurrence of ductal carcinoma in situ after skin-sparing mastectomy. J Am Coll Surg 204:1074-1078

27. Petit JY, Gentilini O, Rotmensz N et al (2008) Oncological results of immediate breast reconstruction: long term follow-up of a large series at a single institution. Breast Cancer Res Treat 112:545-549

28. Khatcheressian JL, Wolff AC, Smith TJ et al (2006) American Society of Clinical Oncology. American Society of Clinical Oncology 2006 update of the breast cancer follow-up and management guidelines in the adjuvant setting. J Clin Oncol 24:5091-5097

29. Caffo O, Cazzolli D, Scalet A et al (2000) Concurrent adjuvant chemotherapy and immediate breast reconstruction with skin expanders after mastectomy for breast cancer. Breast Cancer Res Treat 60:267-275

30. Allweis TM, Boisvert ME, Otero SE et al (2002) Immediate reconstruction after mastectomy for breast cancer does not prolong the time to starting adjuvant chemotherapy. Am J Surg 183:218-221

31. Wilson CR, Brown IM, Weiller-Mithoff E et al (2004) Immediate breast reconstruction does not lead to a delay in the delivery of adjuvant chemotherapy. Eur J Surg Oncol 30:624-627

32. Overgaard M, Hansen PS, Overgaard J et al (1997) Postoperative radiotherapy in high-risk premenopausal women with breast cancer who receive adjuvant chemotherapy. N Engl J Med 337:949-955

33. Ragaz J, Jackson SM, Le N et al (1997) Adjuvant radiotherapy and chemotherapy in node positive premenopausal women with breast cancer. N Engl J Med 337:956-962

34. Kronowitz SJ, Robb GL (2009) Radiation therapy and breast reconstruction: a critical review of the literature. Plast Reconstr Surg 124:395-408

35. Schechter NR, Strom EA, Perkins GH et al (2005) Immediate breast reconstruction can impact postmastectomy radiation. Am J Clin Oncol 28:485-494

36. Buchholz TA, Strom EA, Perkins GH et al (2002) Controversies regarding the use of radiation after mastectomy in breast cancer. Oncologist 7:539-546

37. Tallet AV, Salem N, Moutardier V et al (2003) Radiotherapy and immediate two-stage breast reconstruction with a tissue expander and implant: complications and esthetic results. Int $\mathrm{J}$ Radiat Oncol Biol Phys 57:136-142

38. Pomahac B, Recht A, May JW et al (2006) New trends in breast cancer management: is the era of immediate breast reconstruction changing? Ann Surg 244:282-288

39. Nahabedian MY, Momen B (2008) The Impact of breast reconstruction on the oncologic efficacy of radiation therapy: a retrospective analysis. Ann Plast Surg 60:244-250

40. Bear HD, Anderson S, Brownet A et al (2003) The effect on tumor response of adding sequential preoperative docetaxel to preoperative doxorubicin and cyclophosphamide: preliminary results from National Surgical Adjuvant Breast and Bowel Project Protocol B-27. J Clin Oncol 21:4165-4174

41. Kaufmann M, Hortobagyi GN, Goldhirsch A et al (2006) Recommendations from an international expert panel on the use of neoadjuvant (primary) systemic treatment of operable breast cancer: an update. J Clin Oncol 24:1940-1949

42. Lipa JE, Youssef AA, Kuerer HM, Robb GL, Chang DW (2003) Breast reconstruction in older women: advantages of autogenous tissue. Plast Reconstr Surg 111:1110-1121

43. Pinsolle V, Grinfeder C, Mathoulin-Pelissier S, Faucher A (2006) J Plast Reconstr Aesthet Surg 59:1017-1024

44. Alderman AK, Wilkins EG, Kim HM, Lowery JC (2002) Complications in postmastectomy breast reconstruction: two-year results of the Michigan Breast Reconstruction Outcome Study. Plast Reconstr Surg 109:2265-2274 
45. Michy T, Gimbergues P, Le Bouëdec G et al (2007) What surgical procedure for immediate breast reconstruction after preoperative radiotherapy and chemotherapy? J Chir 144:511-515

46. Spear SL, Onyewu C (2000) Staged breast reconstruction with saline-filled implants in the irradiated breast: recent trends and therapeutic implications. Plast Reconstr Surg 105:930-942

47. Clough KB, Sarfati I, Fitoussi A et al (2005) Breast reconstruction: late cosmetic results of implant reconstruction. Ann Chir Plast Esthet 50:560-574

48. Cordeiro PG, Pusic AL, Disa JJ, McCormick B, VanZee K (2004) Irradiation after immediate tissue expander/implant breast reconstruction: outcomes, complications, aesthetic results, and satisfaction among 156 patients. Plast Reconstr Surg 113:877881

49. Behranwala KA, Dua RS, Ross GM et al (2006) The influence of radiotherapy on capsule formation and aesthetic outcome after immediate breast reconstruction using biodimensional anatomical expander implants. J Plast Reconstr Aesthet Surg 59:1043-1051

50. Contant CM, Van Geel AN, Van der Holt B et al (2000) Morbidity of immediate breast reconstruction (IBR) after mastectomy by subpectorally placed silicone prosthesis: the adverse effect of radiotherapy. Eur J Surg Oncol 26:344-350

51. Disa JJ, McCarthy CM, Mehrara BJ, Pusic AL, Cordeiro PG (2008) Immediate latissimus dorsi/prosthetic breast reconstruction following salvage mastectomy after failed lumpectomy/ irradiation. Plast Reconstr Surg 121:159e-164e

52. Freeman ME, Perdikis G, Sternberg EG, TerKonda SP, Waldorf JC (2006) Latissimus dorsi reconstruction: a good option for patients with failed breast conservation therapy. Ann Plast Surg 57:134-137

53. Spear SL, Boehmler JH, Taylor NS, Prada C (2007) The role of the latissimus dorsi flap in reconstruction of the irradiated breast. Plast Reconstr Surg 119:1-9

54. Kroll SS, Khoo A, Singletary SE et al (1999) Local recurrence after skin-sparing and conventional mastectomy: a 6-year followup. Plast Reconstr Surg 104:421-425
55. Buchanan CL, Dorn PL, Fey J et al (2006) Locoregional recurrence after mastectomy: incidence and outcomes. Am Coll Surg 203:469-474

56. Carreno G, del Casar JM, Corte MD et al (2007) Local recurrence after mastectomy for breast cancer: analysis of clinicopathological, biological and prognostic characteristics. Breast Cancer Res Treat 102:61-73

57. Jatoi I, Proschan MA (2005) Randomized trials of breast-conserving therapy versus mastectomy for primary breast cancer: a pooled analysis of updated results. Am J Clin Oncol 28:289-294

58. Sandelin K, Wickman M, Billgren AM (2004) Oncological outcome after immediate breast reconstruction for invasive breast cancer: a long-term study. The Breast 13:210-218

59. Yildirim E, Berberoglu U (2008) Postmastectomy locoregional recurrence and distant metastasis in breast carcinoma patients. The Breast 17:367-371

60. Group EarlyBreastCancerTrialists'Collaborative (2005) Effects of chemotherapy and hormonal therapy for early breast cancer on recurrence and 15-year survival: an overview of the randomised trials. Lancet 365:1687-1717

61. Spear SL, Boehmler JH, Bogue DP et al (2008) Options in reconstructing the irradiated breast. Plast Reconstr Surg 122:379388

62. Gerber B, Krause A, Reimer T et al (2003) Skin-sparing mastectomy with conservation of the nipple-areola complex and autologous reconstruction is an oncologically safe procedure. Ann Surg 238:120-127

63. Thomson HJ, Potter S, Greenwood RJ et al (2008) A prospective longitudinal study of cosmetic outcome in immediate latissimus dorsi breast reconstruction and the influence of radiotherapy. Ann Surg Oncol 15:1081-1091

64. Mauri D, Pavlidis N, Ioannidis JP (2005) Neoadjuvant versus adjuvant systemic treatment in breast cancer: a meta-analysis. J Natl Cancer Inst 97:188-194 